\title{
Organic Light-Emitting Diodes for Optogenetic Stimulation of Drosophila Larvae
}

\author{
Caroline Murawski ${ }^{1}$, Andrew Morton ${ }^{1}$, Ifor D. W. Samuel ${ }^{1}$, Stefan R. Pulver ${ }^{2}$, and Malte C. Gather ${ }^{1}$ \\ 1 Organic Semiconductor Centre, SUPA, School of Physics and Astronomy, University of St Andrews, North Haugh, St Andrews KY16 9SS, UK \\ 2 School of Psychology and Neuroscience, University of St Andrews, St Mary's Quad, South Street, St Andrews KY16 9JP, UK \\ Authore-mail address:cm337@st-andrews.ac.uk
}

\begin{abstract}
Optogenetics is an emerging method in biology that enables controlling neurons with light. We use organic light-emitting diodes to stimulate neurons in Drosophila larvae and investigate subsequent behavioral changes at different light intensities.

OCIS codes: (170.2945) Medical optics and biotechnology - 1llumination design; (310.6860) Thin film devices and applications; (160.4890) Organic materials
\end{abstract}

\section{Introduction}

Optogenetics is an emerging new method in biology for the non-invasive and timely control of neuronal behaviour with light [1]. Currently, however, only a small number of neurons can be controlled individually so that stimulation of neurons with light still lacks precision compared to the complexity of the brain. To overcome these limitations, we propose to develop a novel light source based on organic light-emitting diodes (OLEDs) that provides unprecedented high-resolution optogenetic control of thousands of neurons at once.

OLEDs are plastic-type organic semiconductors that are sandwiched between two thin-film electrodes (Fig. 1). Compared to conventional light sources, OLEDs can in principle address single neurons and quickly switch between targeted cells by using microscopic patterning of the device. Recently, OLED microdisplays were used to stimulate and study locomotion of light-sensitive algae cells [2] and for optogenetic stimulation of HEK cells [3]. These cells can be stimulated at rather low brightness illumination $\left(\approx 1 \mu \mathrm{W} / \mathrm{cm}^{2}\right)$ which is easily achieved in simple OLED structures. The stimulation of neurons, however, requires considerably higher light intensities in the

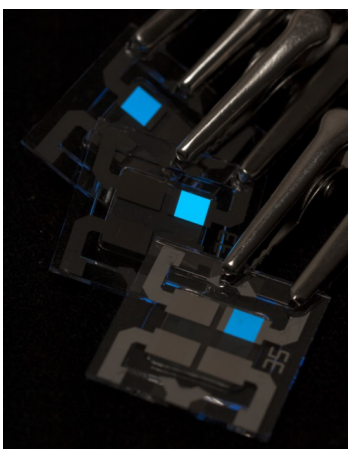

Fig. 1. Photograph of blue OLEDs from our lab range of $0.1-10 \mathrm{~mW} / \mathrm{mm}^{2}$ [4], a brightness at which OLED efficiency typically decreases [5].

In this contribution, we use an efficient fluorescent emitter and doped charge transport layers [6] in order to achieve very high brightness at low voltages. Concurrently, we demonstrate that these high-brightness OLEDs can be used to robustly excite neurons in living Drosophila (fruit fly) larvae.

\section{High-brightness OLEDs}

The structure of the OLEDs used in this study is shown in Fig. 2a. OLEDs are prepared by thermal evaporation in a UHV chamber (Angstrom Engineering) at a base pressure of $10^{-7} \mathrm{mbar}$ on pre-cleaned glass substrates that were prepatterned with ITO. The materials involved are 2,2',7,7'-tetrakis(N,N'-di-p-methylphenylamino)-9,9'spirobifluorene (Spiro-TTB) p-doped with 2,2'-(perfluoronaphthalene-2,6-diylidene)dimalononitrile (F6-TCNNQ) $(2 \mathrm{wt} \%)$ as hole transport layer, N,N'-di(naphtalene-1-yl)-N,N'-diphenylbenzidine (NPB) as electron blocking layer, the fluorescent emitter 2,5,8,11-tetra-tert-butylperylene (TBPe) doped with $1.5 \mathrm{wt} \%$ into the host 2-methyl-9,10bis(naphthalen-2-yl)anthracene (MADN) as emission layer, bis-(2-methyl-8-chinolinolato)-(4-phenyl-phenolato)aluminium(III) (BAlq2) as hole blocking layer, and 4,7-diphenyl-1,10-phenanthroline (BPhen) doped with cesium $(2 \mathrm{wt} \%)$ as n-doped electron transport layer, finished by an aluminum cathode. Samples are encapsulated in nitrogen atmosphere with a glass lid and moisture getter directly after fabrication. The active area of the device is $16.1 \mathrm{~mm}^{2}$.

Figure $2 \mathrm{~b}$ shows the current density and optical power density of the device as a function of the voltage. Due to efficient doping of the charge transport layers, current densities above $1 \mathrm{~A} / \mathrm{cm}^{2}$ are reached at $12 \mathrm{~V}$. We measured the power density using a calibrated power meter (Gentec-EO) with a photodiode (PH100-Si) placed in direct contact with the OLED, while the OLED was driven in steady state (voltage pulse length $>1 \mathrm{~s}$ ). A maximum brightness of $0.85 \mathrm{~mW} / \mathrm{mm}^{2}$ was reached at $14 \mathrm{~V}$, which is sufficient to trigger action potentials in neurons that are transfected with relatively light-sensitive ion channels (e.g. ChR2 C128S [7], CsChrimson [8]). Optimizing the sample layout further, we believe that power densities above $1 \mathrm{~mW} / \mathrm{mm}^{2}$ can be reached even below $10 \mathrm{~V}$ by reducing the voltage loss in ITO contact lines. 
a)

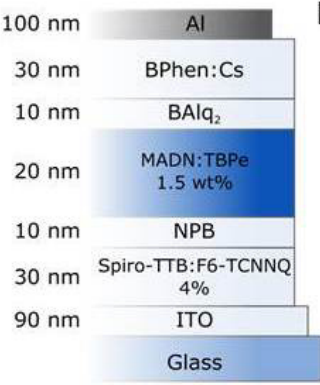

b)

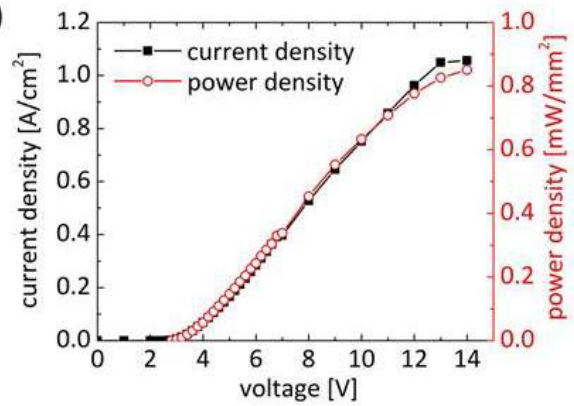

c)

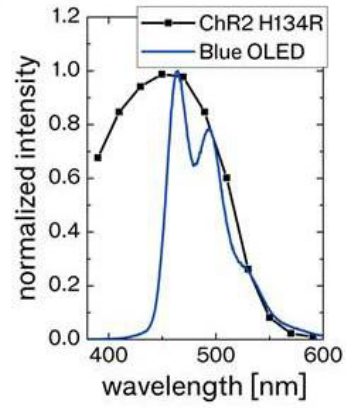

Fig. 2. a) Structure of the OLED. b) Current density and optical power density of the OLED as a function of the voltage. c) OLED emission spectrum and activation spectrum of the channelrhodopsin ChR2 H134R [9].

A key requirement to enable optogenetic activation is a good overlap of the emission spectrum of the light source with the activation spectrum of the used channelrhodopsin. Figure $2 \mathrm{c}$ shows the emission spectrum of the OLED in forward direction. The emission shows two peaks at $465 \mathrm{~nm}$ and $493 \mathrm{~nm}$ and the maximum of the spectrum is located at the maximum of the activation spectrum of the channelrhodopsin ChR2 H134R [9]. In addition, the relatively broad spectrum of the OLED overlaps very well with the activation spectrum of the ChR making the OLED highly suitable for optogenetic activation.

\section{Stimulating neurons in live animals}

In order to test that the brightness of our OLEDs is sufficient to trigger action potentials in neurons, we used the devices to stimulate living Drosophila larvae that were expressing the channelrhodopsin ChR2 H134R in motor neurons. Stimulation of the larvae with blue light thus leads to a firing of action potentials in motor neurons, which causes a visible contraction and immobilization of the larva $[10,11]$.

OK371-GAL4/UAS-H134R-ChR2 flies were raised on solid cornmeal-based medium supplemented with $1 \mathrm{mM}$ all-trans-retinal. We mounted the OLED underneath an upright microscope (Nikon Eclipse Ni) with 4x/0.13 NA objective and studied the behavior of third instar larvae by confining them in a silicone chamber filled with sucrose solution $(5 \% \mathrm{w} / \mathrm{v})$, which was located on top of the OLED. The sample was illuminated by a fibre-coupled mercury light source attenuated with ND filters to $<1 \mathrm{~mW} / \mathrm{mm}^{2}$ and long pass filtered $(>600 \mathrm{~nm})$ in order to avoid activation of the ChR. The OLED was driven with voltage pulses of $5 \mathrm{~s}$ duration and $50 \%$ duty cycle $(0.1 \mathrm{~Hz}$ frequency). The driving voltage of the OLED was varied in order to study the larval response to different brightness levels. The larval behavior was measured with an attached sCMOS camera (Andor Neo) over the course of $60 \mathrm{~s}$ at a speed of 10 frames/s.

Exemplary images of the larva on top of the OLED are shown in Fig. 3a. The larva was relaxed if the OLED was turned off but showed a strong contraction when the OLED was turned on. In order to quantify the larval response, we tracked the positions of head and tail in each frame of the recorded traces using the software Image J and the plugin MtrackJ. From those positions, we calculated the distance between head and tail, which indicates whether the larva is relaxed or contracted. OLED illumination timing was tracked from the videos as well. As an example, Figure 3b shows head-tail distance traces for two OLED power densities, marking the OLED on-time by blue shaded areas. Upon OLED turn-on, a robust contraction of the larva is observed, while the larva returns to its relaxed state within less than $1 \mathrm{~s}$ after the OLED is turned off. This behavior is achieved at power densities of $10 \mu \mathrm{W} / \mathrm{mm}^{2}$ or above, whereas lower power densities caused no visible contraction.

In order to verify that the contractions are caused by the OLED light source, we calculated the Fourier transform of the head-tail distance traces. The result is shown in Fig. 3c. For power densities from $10 \mu \mathrm{W} / \mathrm{mm}^{2}$, a clear peak in amplitude is observed at $0.1 \mathrm{~Hz}$, the frequency used for switching the OLED. In contrast, lower stimulation brightness causes no observable peak in the Fourier transform, confirming that the light from the OLED did not lead to action potential firing. 
a) $10 \mu \mathrm{W} / \mathrm{mm}^{2}$

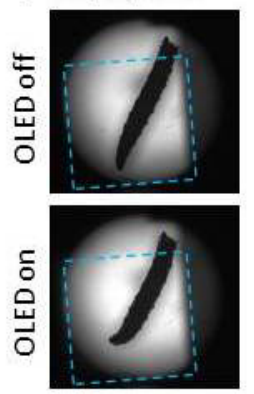

$150 \mu \mathrm{W} / \mathrm{mm}^{2}$

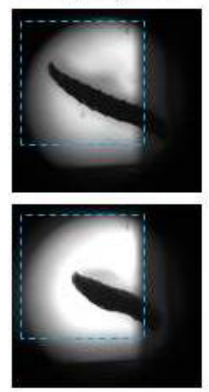

b)

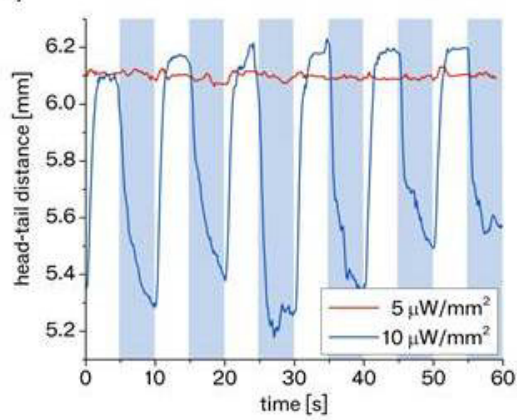

c)

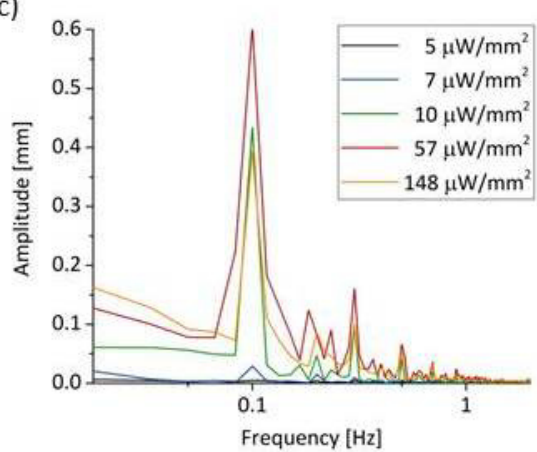

Fig. 3. Larval response upon stimulation with $5 \mathrm{~s}$ long light pulses at different optical powers. a) Image of the larva on top of the OLED. Top: OLED off. Bottom: OLED on. Blue dashed lines indicate the OLED pixel area. b) Head-tail distance traces. Blue shaded areas mark when the OLED was turned on. A robust contraction of the larva is detected for power densities from $10 \mu \mathrm{W} / \mathrm{mm}^{2}$. c) Fourier-transform of the head-tail distance traces marking a peak response at the stimulation frequency of $0.1 \mathrm{~Hz}$.

\section{Conclusions}

In this contribution, we demonstrated that OLEDs are capable of stimulating neurons in living animals. In particular, we have proven that OLEDs can achieve high enough brightness to trigger robust action potential firing. We envisage that the brightness of our OLEDs can be further increased by reducing resistive losses in the ITO contacts and by reducing the pixel area in order to decrease losses due to Joule heating.

\section{Acknowledgment}

We are grateful for financial support from the Scottish Funding Council (through SUPA), Human Frontier Science Program (RGY0074/2013), Wellcome Trust Institutional Strategic Support Fund St Andrews, the RS Macdonald Charitable Trust, and EPSRC via grant EP/J01771X/1. CM acknowledges funding by the European Commission through a Marie Skłodowska Curie individual fellowship (703387).

\section{References}

1. E. S. Boyden, F. Zhang, E. Bamberg, G. Nagel, and K. Deisseroth, "Millisecond-timescale, genetically targeted optical control of neural activity," Nat. Neurosci. 8, 1263-1268 (2005).

2. A. Steude, M. Jahnel, M. Thomschke, M. Schober, and M. C. Gather, "Controlling the Behavior of Single Live Cells with High Density Arrays of Microscopic OLEDs," Adv. Mater. 27, 7657-7661 (2015).

3. A. Steude, E. C. Witts, G. B. Miles, and M. C. Gather, "Arrays of microscopic organic LEDs for high-resolution optogenetics," Sci. Adv. 2, e1600061 (2016).

4. O. Yizhar, L. E. Fenno, T. J. Davidson, M. Mogri, and K. Deisseroth, "Optogenetics in Neural Systems," Neuron 71, 9-34 (2011).

5. C. Murawski, K. Leo, and M. C. Gather, "Efficiency Roll-Off in Organic Light-Emitting Diodes," Adv. Mater. 25, 6801 (2013).

6. K. Walzer, B. Maennig, M. Pfeiffer, and K. Leo, "Highly efficient organic devices based on electrically doped transport layers," Chem. Rev. 107, 1233-71 (2007).

7. A. Berndt, O. Yizhar, L. A. Gunaydin, P. Hegemann, and K. Deisseroth, "Bi-stable neural state switches.," Nat. Neurosci. 12, 229-234 (2009).

8. N. C. Klapoetke, Y. Murata, S. S. Kim, S. R. Pulver, A. Birdsey-Benson, Y. K. Cho, T. K. Morimoto, A. S. Chuong, E. J. Carpenter, Z. Tian, J. Wang, Y. Xie, Z. Yan, Y. Zhang, B. Y. Chow, B. Surek, M. Melkonian, V. Jayaraman, M. Constantine-Paton, G. K.-S. Wong, and E. S. Boyden, "Independent optical excitation of distinct neural populations.," Nat. Methods 11, 338-46 (2014).

9. J. Y. Lin, M. Z. Lin, P. Steinbach, and R. Y. Tsien, "Characterization of engineered channelrhodopsin variants with improved properties and kinetics," Biophys. J. 96, 1803-1814 (2009).

10. S. R. Pulver, N. J. Hornstein, B. L. Land, and B. R. Johnson, "Optogenetics in the teaching laboratory: using channelrhodopsin-2 to study the neural basis of behavior and synaptic physiology in Drosophila," AJP Adv. Physiol. Educ. 35, 82-91 (2011).

11. S. R. Pulver, S. L. Pashkovski, N. J. Hornstein, P. a Garrity, and L. C. Griffith, "Temporal dynamics of neuronal activation by Channelrhodopsin-2 and TRPA1 determine behavioral output in Drosophila larvae.," J. Neurophysiol. 101, 3075-3088 (2009). 